\title{
Thickness conditions for characterizing the periodic nanostructures with the retrieved electromagnetic parameters
}

\section{Song}

\section{Z. Tang \\ txz@hnu.edu.cn}

\section{Zhao}

Z. Sui

\section{S. Wen}

scwen@hnu.edu.cn

D. Fan
Key Laboratory for Micro-/Nano-Optoelectronic Devices of Ministry of Education, College of Information Science and Engineering, Hunan University, Changsha 410082, People's Republic of China

Key Laboratory for Micro-/Nano-Optoelectronic Devices of Ministry of Education, College of Information Science and Engineering, Hunan University, Changsha 410082, People's Republic of China

Research Center of Laser Fusion, Chinese Academic of Engineering Physics, Mianyang 621900, People's Republic of China

Research Center of Laser Fusion, Chinese Academic of Engineering Physics, Mianyang 621900, People's Republic of China

Key Laboratory for Micro-/Nano-Optoelectronic Devices of Ministry of Education, College of Information Science and Engineering, Hunan University, Changsha 410082, People's Republic of China

Key Laboratory for Micro-/Nano-Optoelectronic Devices of Ministry of Education, College of Information Science and Engineering, Hunan University, Changsha 410082, People's Republic of China

By analyzing the convergence of the retrieved effective electromagnetic parameters, we presented that one wavelength of the propagating wave in the nanostructure is the minimum thickness requirement for effectively characterizing a finite thickness nanostructure. This thickness condition has been separately validated in a photonic crystal with negative refraction and in a typical fishnet metamaterial which has been investigated theoretically and experimentally before.

[DOI: http://dx.doi.org/10.2971/jeos.2013.13028]

Keywords: Photonic crystal, retrieved electromagnetic parameters, negative refraction

\section{INTRODUCTION}

Periodic nanostructures, such as photonic crystals (PCs) and metamaterials, are often characterized by the effective electromagnetic parameters based on implicit assumptions inspired by natural material models [1]. In most cases, these effective electromagnetic parameters are obtained by utilizing the field averaging [2]-[6], extended Maxwell-Garnett [7]-[10] or S-parameter retrieval method [11]-[20]. However, different from bulk homogeneous material, the retrieved parameters of nanostructures, especially the fishnet metamaterials, are always sensitive to the slab thicknesses [21]-[24]. Therefore, in this respect, most experimental samples such as the stacking of three-functional [25], four-functional [26], and tenfunctional [27, 28] layers and even the thickest fabricated fishnet structures cannot be treated as a homogeneous metamaterial with effective electromagnetic parameters.

Although we have known that these parameters will be converged and independent of the slab thicknesses when the slab thickness increases to some extent [21]-[24], it is still unclear what is the minimum thickness prerequisite for achieving this convergence and what is the underlying physics. In this paper, we focus on tackling these problems. Unlike the works on the convergence of retrieved parameters in fishnet metamaterials, we take a PC with a negative refractive index as an example, and systematically investigate the dependence of the re- trieved effective electromagnetic parameters on the distances between the input/output port and the PC slab. Nevertheless, we will borrow the similar phenomena discussed intensively in fishnet metamaterials to analyze the physics underlying the thickness conditions. Particularly, we will establish guidelines for the thickness conditions under which the periodic nanostructures such as PCs and metamaterials can be characterized by the effective electromagnetic parameters.

\section{MODEL AND METHOD}

The two-dimensional (2D) dielectric PC slab with a negative refractive index we take as an example is shown in Figure 1(a). This PC structure is made of a square lattice of air holes etched in a low loss material with dielectric permittivity constant $\varepsilon=10.6$ [29]. The lattice constant is $a$ and the diameter of the air hole is $2 r=0.7 a$. Throughout this paper we only consider the transverse-magnetic (TM) modes whose electric field is polarized along the air holes.

Using the plane wave expansion method, the band structure of this PC is calculated and shown in Figure 1(b), where the frequency $\left(f_{\text {nor }}\right)$ is normalized to $a / \lambda_{0}\left(\lambda_{0}\right.$ is the wavelength in vacuum). As shown in Figure 1(b), a left-handed dispersion branch can be noted in the second band. The normalized fre- 
(a)

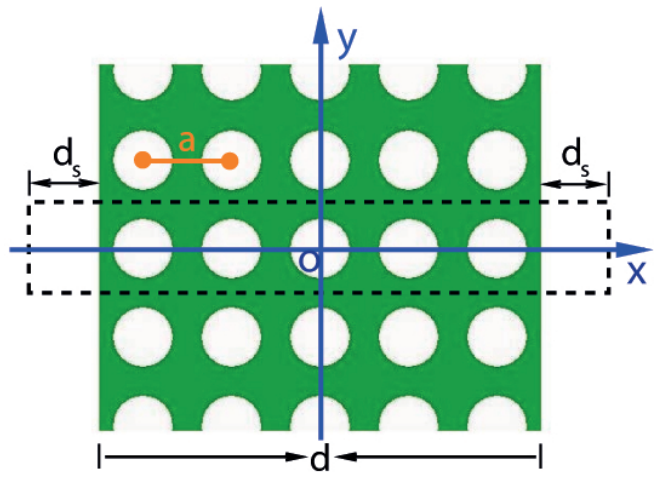

(b)

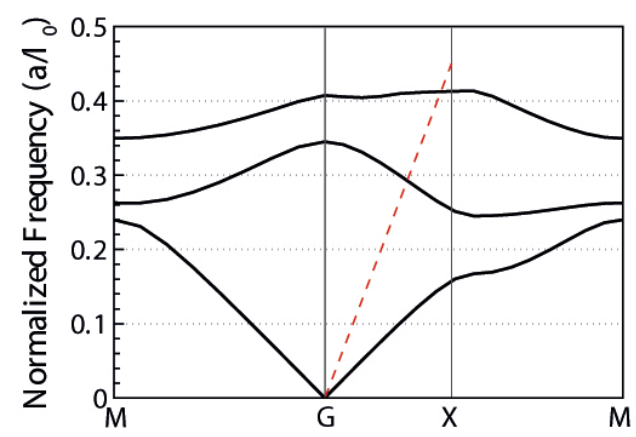

FIC. 1 (a) Schematic of the PC and the coordinate system describing simulation processes. (b) Band structure of the PC for the TM polarization. The dashed line denotes the light line in vacuum.

quency $f_{\text {nor }}=0.2858$, at which the effective index $n_{\text {eff }}=-1$, is denoted by the intersection point between the light line and the second band along the $\Gamma-X$ direction. With $a=443 \mathrm{~nm}$, this normalized frequency corresponds to the telecommunication wavelength $\lambda=1550 \mathrm{~nm}$.

Although the PCs are beyond the traditional long wavelength limit [8], they can be described by the effective parameters under the single-mode approximation [30, 31]. For electromagnetic waves incident normally to the PC surfaces, the effective refractive index $n_{\text {eff }}$ and effective impedance $z_{\text {eff }}$ are related to the reflection coefficient $S_{11}$ at the front interface and the transmission coefficient $S_{21}$ at the back interface by [11]

$$
\begin{gathered}
n_{\mathrm{eff}}=\frac{1}{k_{0} d} \arccos \left[\frac{1}{2 S_{21}}\left(1-S_{11}^{2}+S_{21}^{2}\right)\right]+\frac{2 m \pi}{k_{0} d}, \\
z_{\mathrm{eff}}= \pm \sqrt{\frac{\left(1+S_{11}\right)^{2}-S_{21}^{2}}{\left(1-S_{11}\right)^{2}-S_{21}^{2}}}
\end{gathered}
$$

where $m$ is an integer related to the branch index of the real part of effective refractive index $\operatorname{Re}\left(n_{\text {eff }}\right), d$ is the slab thickness, and $k_{0}$ is the wave number of the incident wave in free space. Since the PC is a passive medium, the corresponding real part of effective impedance $\operatorname{Re}\left(z_{\text {eff }}\right)$ and imaginary part of effective refractive index $\operatorname{Im}\left(n_{\text {eff }}\right)$ must be greater than zero according to the causality. This retrieval procedure is initially presented by Weir [32], and now has been successfully applied to periodic nanostructures [11]-[20].

The transmission and reflection coefficients, used in the retrieval procedure, are obtained by using the finite-difference time-domain (FDTD) method [33] in this paper. Periodic boundary conditions are employed to simulate an infinite lattice perpendicular to the direction of propagation, and per-

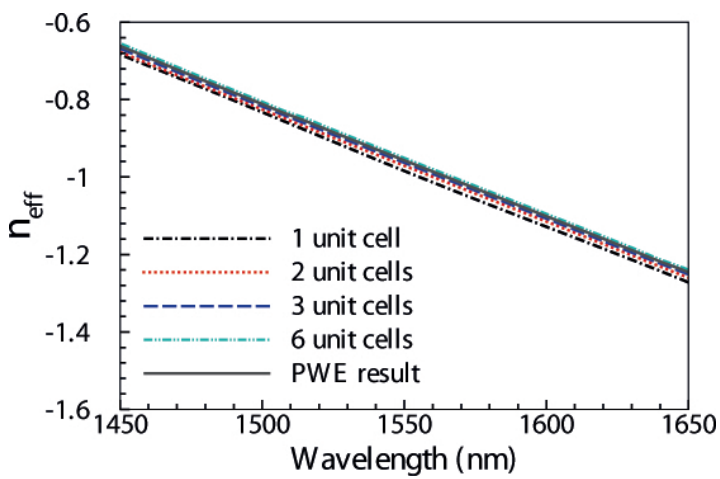

FIG. 2 Retrieved effective refractive index $n_{\text {eff }}$ with $d_{\mathrm{s}}=0 \mathrm{~nm}$ for different number of unit cells.

fectly matched layers are used for the remaining boundaries. Along the propagation direction, two recorders are located at at $d_{S}$ away from the PC interfaces to record $S_{11}^{\prime}$ and $S_{21}^{\prime}$, respectively. Then, the $S$ parameters at the slab interfaces, which are used in the retrieval procedure, can be obtained by $S_{11}=S_{11}^{\prime} \exp \left(-i k_{0} 2 d_{\mathrm{s}}\right)$ and $S_{21}=S_{21}^{\prime} \exp \left(-i k_{0} 2 d_{\mathrm{s}}\right)$. With this kind of configuration, the finite-thickness nanostructures cannot be treated as a homogeneous material if the retrieved parameters are dependent on either $d_{\mathrm{s}}$ or the thicknesses.

\section{RESULTS AND DISCUSSION}

To check these dependence of our photonic crystal samples, we first calculated the effective refractive index $n_{\text {eff }}$ as a function of wavelength $\lambda$ for one unit cell, two, three and six unit cells of PC with $d_{\mathrm{s}}=0 \mathrm{~nm}$. For comparison, the $n_{\text {eff }}$ of the infinite PC has also been figured out by solving the master equation using the plane wave expansion (PWE) method. As shown in Figure 2, all curves of the $n_{\text {eff }}$ almost overlapped each other and the $n_{\text {eff }}$ increases monotonously with $\lambda$ from about -1.25 to -0.65 . These results indicated that for all retrieved models with $d_{\mathrm{s}}=0 \mathrm{~nm}$, irrespective of the numbers of unit cells, mimic very closely the infinite periodic nanostructure. Just for this reason, this configuration with $d_{\mathrm{s}}=0 \mathrm{~nm}$ has been widely used for electromagnetic characterizing the infinite nanostructures. However, most of the fabricated samples at optical wavelengths were only a few layers. Are the effective parameters of the infinite nanostructures still suitable for such thin samples?

In fact, there are complicated surface waves generated at the interfaces between the periodic nanostructure and the free space. For a very thin nanostructure, the surface waves at the front interface always extend to the back one. As a result, the retrieved effective electromagnetic parameters will vary with $d_{\mathrm{s}}$ till the effects of the surface waves can be ignored.

To avoid the effects of the surface waves, we adopted configurations with $d_{\mathrm{s}} \neq 0 \mathrm{~nm}$ (this kind of configuration is widely used to avoid the numerical errors for calculating $S$ parameters at the inhomogeneous interfaces). As we known the surface waves fade out exponentially in the free space, so we use a $d_{\mathrm{s}}$ around $\lambda_{0}$ to diminish the effects of surface waves $\left(\lambda_{0}\right.$ is the wavelength in free space). 
In this paper, $d_{\mathrm{S}}=1550 \mathrm{~nm}$ has been taken as an example, and the retrieved parameters have been recalculated and plotted in Figure 3. Compared with the results of $d_{\mathrm{s}}=0 \mathrm{~nm}$, it is obvious that different parameters have been obtained for one unit cell and two unit cells. But when the number of unit cells is larger than three, the retrieved $n_{\text {eff }}$ nearly all overlapped those of the infinite periodic nanostructures again. Additionally, similar phenomena have also been found in Figure 3(b) for the other retrieved electromagnetic parameter, i.e., the effective impedance $z_{\text {eff }}$. Because of the same retrieved results, the retrieved results of 4 and 5 unit cells have been omitted from Figure 3 for the sake of clarity. From the above results, we can conclude that for this PC near $f_{\text {nor }}=0.2858$ three-unitcells thick is the minimum thickness requirement for achieving converged retrieved parameters.

Definitely, the convergency of the retrieved parameters are determined by the resonant interactions between the electromagnetic waves and the periodic structures, which is the

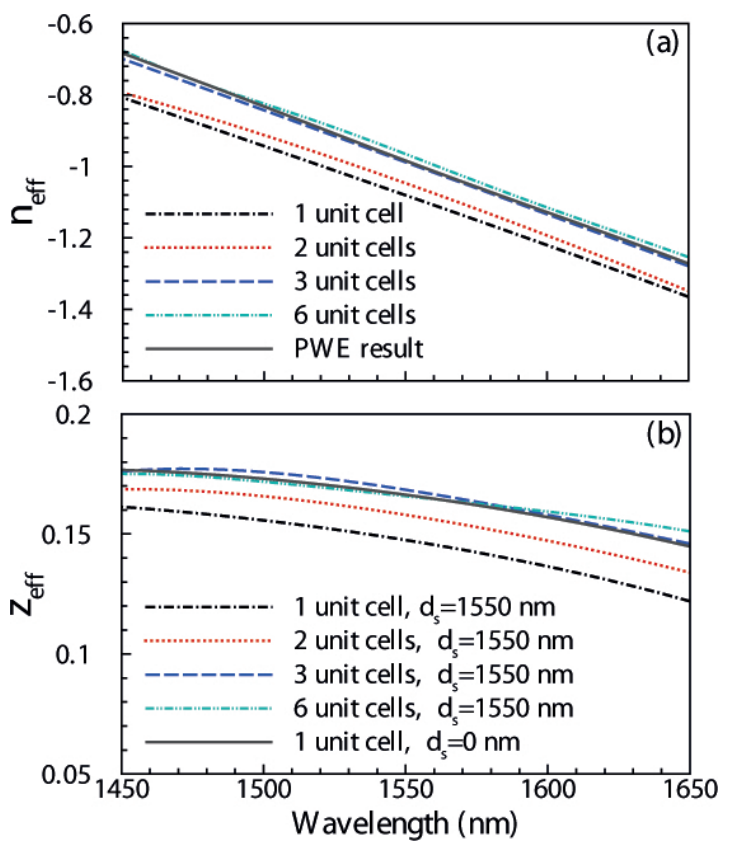

FIG. 3 (a) Retrieved effective refractive index $n_{\text {eff }}$ and (b) impedance $z_{\text {eff }}$ with $d_{\mathrm{s}}=1550 \mathrm{~nm}$ for different number of unit cells. The $n_{\text {eff }}$ obtained by utilizing the PWE method and the $z_{\text {eff }}$ with $d_{\mathrm{s}}=0 \mathrm{~nm}$ for one unit cell are added to (a) and (b), respectively.

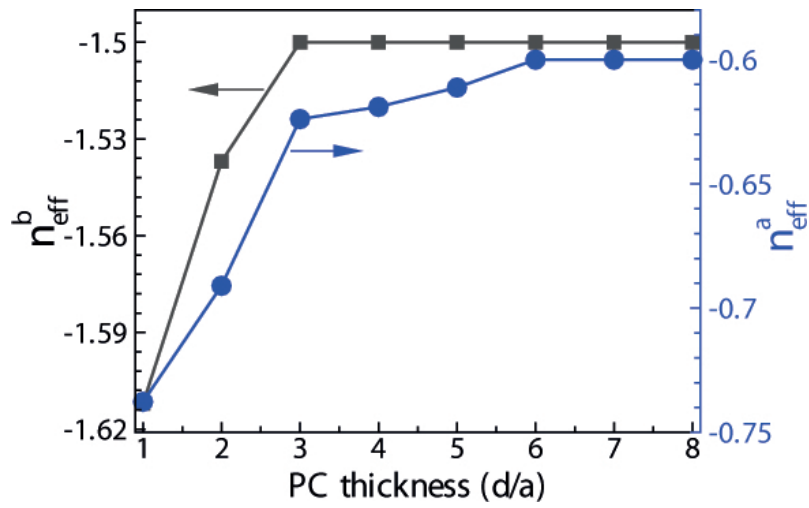

FIG. 4 Retrieved effective refractive index $n_{\text {eff }}$ as the function of the PC thickness with $d_{\mathrm{s}}=1550 \mathrm{~nm}$. physical origin of the abnormal dispersion of PCs, To establish these interactions basically, the periodic nanostructures should be thick enough to hold nearly one whole oscillation. In other words, the nanostructures' thicknesses at least are close to the wavelengthes of the propagating waves in the nanostructure. This suggests a thickness condition under which the retrieved effective electromagnetic parameters can be used to characterize the finite periodic nanostructures.

To verify the above deduction, we analyzed the dependence of the retrieved effective refractive index $n_{\text {eff }}$ on the layer thickness with $d_{\mathrm{s}}=1550 \mathrm{~nm}$ for another two normalized frequencies, i.e., $f_{\text {nor }}^{a}=0.32$ and $f_{\text {nor }}^{b}=0.263$. As shown in Figure 4, for $f_{\text {nor }}^{a}=0.32$ the retrieved refractive index converges to $n_{\text {eff }}^{a}=-0.6$ when the slab is thicker than $5 a$. Moreover, the wavelength in PC slab at this frequency is $\lambda_{a}=a / f_{\text {nor }}^{a}\left|n_{\text {eff }}^{a}\right|=5.21 a$. For $f_{\text {nor }}^{b}=0.263$, the minimum thickness for achieving the convergence of $n_{\text {eff }}^{b}$ changes to $3 a$ and the wavelength in PC slab is $\lambda_{b}=a / f_{\text {nor }}^{b}\left|n_{\text {eff }}^{b}\right|=2.53 a$. It is obvious that the retrieved parameters converge when the slab thickness is close to the wavelength of the propagating wave in the PCs.

In addition, similar calculations were performed to check our assumptions in the fishnet metamaterials. We took the same model discussed in the Refs. [22] and [28]. Due to its inhomogeneous interfaces, these two recorders were placed far away from the interfaces of the fishnet metamaterial. The retrieved effective refractive index $n_{\text {eff }}$ as a function of the layer numbers were plotted in Figure 5. For the wavelength in vacuum $\lambda_{c}=1811 \mathrm{~nm}$, the retrieved $n_{\text {eff }}^{c}$ waves with the layer numbers at first and converges to -2.0 at last when the thickness of the fishnet MMs exceeds $11 a_{\mathrm{z}}$, where $a_{\mathrm{z}}=2 t+s(t$ and $s$ correspond to the thickness of metallic cladding (Ag) and dielectric spacer $\left.\left(\mathrm{MgF}_{2}\right)\right)$ is the unit thickness. For this model, $a_{\mathrm{z}}=80 \mathrm{~nm}$ and $11 a_{\mathrm{z}}=880 \mathrm{~nm} \approx \lambda_{c} /\left|n_{\mathrm{eff}}^{c}\right|$. The same thing takes place for the wavelength in vacuum $\lambda_{d}=1763 \mathrm{~nm}$ whose retrieved $n_{\text {eff }}^{d}$ are converged to -1.55 until the layer numbers

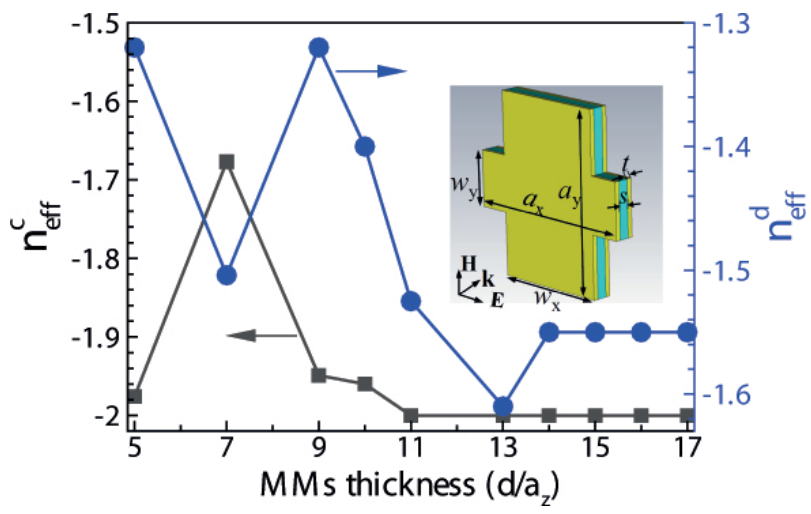

FIG. 5 Retrieved effective refractive index $n_{\text {eff }}$ as the function of the fishnet metamaterials (MMs) thickness with $d_{\mathrm{s}}=1811 \mathrm{~nm}$ for $n_{\mathrm{eff}}^{c}=-2.0$ and $d_{\mathrm{s}}=1763 \mathrm{~nm}$ for $n_{\text {eff }}^{d}=-1.55$. Inset shows the MMs design and polarization configuration. The geometric parameters are $a_{\mathrm{x}}=a_{\mathrm{y}}=860 \mathrm{~nm}, w_{\mathrm{x}}=565 \mathrm{~nm}, w_{\mathrm{y}}=265 \mathrm{~nm} . t=15 \mathrm{~nm}$ and $s=50 \mathrm{~nm}$ are corresponding to the thickness of metallic cladding (Ag) and dielectric spacer $\left(\mathrm{MgF}_{2}\right)$. A Drude model is used for the dielectric parameters of silver, with plasma frequency $\omega_{p}=9.0 \mathrm{eV}$ and scatting frequency $\gamma=0.054 \mathrm{eV}$. The dielectric constant of the $\mathrm{MgF}_{2}$ is $\epsilon_{r}=1.9$. 
are larger than $\lambda_{d} /\left|n_{\text {eff }}^{d}\right| / a_{\mathrm{z}} \approx 14$. We should mention here that, although the lattice constant of the fishnet metamaterial along the light propagation direction is far less than the wavelength, the others are of the same order of magnitude to the wavelength [34]-[36].

\section{CONCLUSION}

In summary, we have presented a thickness condition under which the periodic nanostructures, such as PCs and metamaterials, can be characterized by the effective electromagnetic parameters within the single-Bloch-mode approximation. This thickness condition, which has been demonstrated in a photonic crystal and in a typical fishnet metamaterial respectively, will be helpful for the design of ultrathin periodic nanostructure-based optical devices.

Although we have only discussed a specific square lattice PC and fishnet metamaterial, this conclusion can be extended to other nanostructures with abnormal dispersion. However, we should point out that the thickness condition is unsuitable for the nanostructures in the traditional long-wavelength limit due to different physical origins.

\section{ACKNOWLEDGEMENTS}

This work is partially supported by the National Basic Research Program (973 Program) of China (Grant No. 2012CB315701), the Natural Science Foundation of China (Grant Nos. 61025024 and 11076011), and Hunan Provincial Natural Science Foundation of China (Grant No. 12JJ7005).

\section{References}

[1] A. Alù, "Restoring the physical meaning of metamaterial constitutive parameters," Phys. Rev. B 83,081102(R) (2011).

[2] R. Biswas, Z. Y. Li, and K. M. Ho, "Impedance of photonic crystals and photonic crystal waveguides," Appl. Phys. Lett. 84, 1254 (2004).

[3] D. R. Smith, and J. B. Pendry, "Homogenization of metamaterials by field averaging (invited paper)," J. Opt. Soc. Am. B 23, 391-403 (2006).

[4] B. Momeni, A. A. Eftekhar, and A. Adibi, "Effective impedance model for analysis of reflection at the interfaces of photonic crystals," Opt. Lett. 32, 778-780 (2007).

[5] B. Momeni, M. Badieirostami, and A. Adibi, “Accurate and efficient techniques for the analysis of reflection at the interfaces of threedimensional photonic crystals," J. Opt. Soc. Am. B 24, 2957-2963 (2007).

[6] Z. L. Lu, and D. W. Prather, "Calculation of effective permittivity, permeability, and surface impedance of negative-refraction photonic crystals," Opt. Express 15, 8340-8345 (2007).

[7] V. Yannopapas, and A. Moroz, "Negative refractive index metamaterials from inherently non-magnetic materials for deep infrared to terahertz frequency ranges," J. Phys.: Condens. Matter 17, 3717 (2005).
[8] Y. Wu, J. Li, Z.-Q. Zhang, and C. T. Chan, "Effective medium theory for magnetodielectric composites: Beyond the long-wavelength limit," Phys. Rev. B 74, 085111 (2006).

[9] V. Yannopapas, "Negative refraction in random photonic alloys of polaritonic and plasmonic microspheres," Phys. Rev. B 75, 035112 (2007).

[10] X. Huang, Y. Lai, Z. H. Hang, H. Zheng, and C. T. Chan, "Dirac cones induced by accidental degeneracy in photonic crystals and zero-refractive-index materials," Nat. Mater. 10, 582-586 (2011).

[11] D. R. Smith, S. Schultz, P. Markoš, and C. M. Soukoulis, “Determination of effective permittivity and permeability of metamaterials from reflection and transmission coefficients," Phys. Rev. B 65 , 195104 (2002).

[12] S. O'Brien, and J. B. Pendry, "Photonic band-gap effects and magnetic activity in dielectric composites," J. Phys.: Condens. Matter 14, 4035 (2002).

[13] X. Chen, T. M. Grzegorczyk, B.-I. Wu, J. Pacheco, Jr., and J. A. Kong, "Robust method to retrieve the constitutive effective parameters of metamaterials," Phys. Rev. E 70, 016608 (2004).

[14] D. R. Smith, D. C. Vier, Th. Koschny, and C. M. Soukoulis, "Electromagnetic parameter retrieval from inhomogeneous metamaterials," Phys. Rev. E 71, 036617 (2005).

[15] C. R. Simovski, "Bloch material parameters of magneto-dielectric metamaterials and the concept of Bloch lattices," Metamaterials 1, 62-80 (2007).

[16] C. R. Simovski and S. A. Tretyakov, "Local constitutive parameters of metamaterials from an effective-medium perspective," Phys. Rev. B 75, 195111 (2007).

[17] C. Croënne, N. Fabre, D. P. Gaillot, O. Vanbésien, and D. Lippens, "Bloch impedance in negative index photonic crystals," Phys. Rev. B 77, 125333 (2008).

[18] C. R. Simovski, "Material parameters of metamaterials," Opt. Spectrosc. 107, 726-753 (2009).

[19] C. Tserkezis and N. Stefanou, "Retrieving local effective constitutive parameters for anisotropic photonic crystals," Phys. Rev. B 81, 115112 (2010).

[20] X.-X. Liu, D. A. Powell, and A. Alù, "Correcting the Fabry-Perot artifacts in metamaterial retrieval procedures," Phys. Rev. B 84, 235106 (2011).

[21] J. F. Zhou, T. Koschny, M. Kafesaki, and C. M. Soukoulis, "Size dependence and convergence of the retrieval parameters of metamaterials," Photonics Nanostruct. Fundam. Appl. 6, 96-101 (2008).

[22] J. F. Zhou, T. Koschny, M. Kafesaki, and C. M. Soukoulis, "Negative refractive index response of weakly and strongly coupled optical metamaterials," Phys. Rev. B 80, 035109 (2009).

[23] W. C. Chen, A. Totachawattana, K. Fan, J. L. Ponsetto, A. C. Strikwerda, X. Zhang, R. D. Averitt, and W. J. Padilla, "Single-layer terahertz metamaterials with bulk optical constants," Phys. Rev. B 85, 035112 (2012).

[24] S. Engelbrecht, A. M. Shuvaev, Ch. Kant, K. Unterrainer, and A. Pimenov, "Experimental determination of effective parameters in a layered metamaterial," Phys. Rev. B 85, 235437 (2012).

[25] G. Dolling, M. Wegener, and S. Linden, "Realization of a threefunctional-layer negative-index photonic metamaterial," Opt. Lett. 32, 551-553f (2007).

[26] N. Liu, H. Guo, L. Fu, S. Kaiser, H. Schweizer, and H. Giessen, "Three-dimensional photonic metamaterials at optical frequencies," Nat. Mater. 7, 31-37 (2008). 
[27] N. Katsarakis, G. Konstantinidis, A. Kostopoulos, S. R. Penciu, T. F. Gundogdu, M. Kafesaki, E. N. Economou, et al., "Magnetic response of split-ring resonators in the far-infrared frequency regime," Opt. Lett. 30, 1348-1350 (2005).

[28] J. Valentine, S. Zhang, T. Zentgraf, E. Ulin-Avila, D. A. Genov, G. Bartal, and X. Zhang, "Three-dimensional optical metamaterial with a negative refractive index," Nature 455, 376-379 (2008).

[29] N. Fabre, L. Lalouat, B. Cluzel, X. Mélique, D. Lippens, F. de Fornel, and 0. Vanbésien, "Optical Near-Field Microscopy of Light Focusing through a Photonic Crystal Flat Lens," Phys. Rev. Lett. 101, 073901 (2008).

[30] W. Śmigaj and B. Gralak, "Validity of the effective-medium approximation of photonic crystals," Phys. Rev. B 77, 235445 (2008).

[31] A. Andryieuski, S. Ha, A. A. Sukhorukov, Y. S. Kivshar, and A. V. Lavrinenko, "Bloch-mode analysis for retrieving effective parameters of metamaterials," Phys. Rev. B 86, 035127 (2012).
[32] W. B. Weir, "Automatic measurement of complex dielectric constant and permeability at microwave frequencies," Proc. IEEE 62, 33-36 (1974).

[33] Dong Jun Technology, EastFDTD v3.0, (Dongjun Information Technology Co., Shanghai, 2011).

[34] C. Fietz, Y. Urzhumov, and G. Shvets, "Complex k band diagrams of 3D metamaterial/photonic crystals," Opt. Express 19, 19027-19041 (2011).

[35] J. A. Reyes-Avendaño, U. Algredo-Badillo, P. Halevi, and F. PérezRodríguez, "From photonic crystals to metamaterials: the bianisotropic response," New J. Phys. 13, 073041 (2011).

[36] P. Y. Chen, C. G. Poulton, A. A. Asatryan, M. J. Steel, L. C. Botten, C. M. de Sterke, and R. C. McPhedran, "Folded bands in metamaterial photonic crystals," New J. Phys. 13, 053007 (2011). 